International Journal of Applied Dental Sciences 2021; 7(1): 245-250

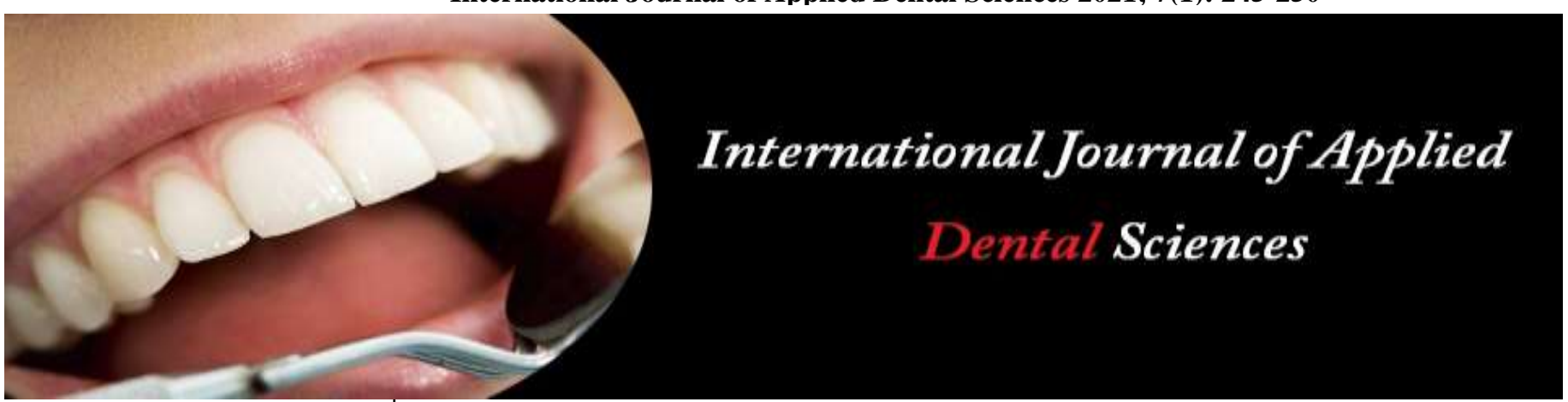

ISSN Print: 2394-7489

ISSN Online: 2394-7497

IJADS 2021; 7(1): 245-250

(C) 2021 IJADS

www.oraljournal.com

Received: 28-10-2020

Accepted: 08-12-2020

Somya Jain

MDS, Department of

Conservative Dentistry and

Endodontics, Surendera Dental

College and Research Institute,

Sri Ganaganagar, Rajasthan,

India

\section{Neetu Jindal}

Professor and Head of the

Department of Conservative

Dentistry and Endodontics,

Surendera Dental College and

Research Institute, Sri

Ganaganagar, Rajasthan, India

Renu Agarwal

Professor of the Department of

Conservative Dentistry and

Endodontics, Surendera Dental

College and Research Institute,

Sri Ganaganagar, Rajasthan,

India

\section{Neha Mittal}

MDS, Department of

Conservative Dentistry and

Endodontics, Mahatma Gandhi

Dental College and Hospital,

Jaipur, Rajasthan, India
Corresponding Author:

Somya Jain

MDS, Department of

Conservative Dentistry and

Endodontics, Surendera Dental

College and Research Institute,

Sri Ganaganagar, Rajasthan,

India

\section{Modern perspective of indirect restorations: Two case reports}

\section{Somya Jain, Neetu Jindal, Renu Agarwal and Neha Mittal}

DOI: https://doi.org/10.22271/oral.2021.v7.i1d.1140

\section{Abstract}

The development of digital dentistry has tremendously revolutionized the way restorative procedures are performed today. Computer-Aided Design/Computer-Aided Milling (CAD/CAM) is an integral part of modern dentistry since its inception in the 1980s when CEREC was first introduced 15 years ago. Improved mechanical retention, better marginal integrity and enhanced aesthetics are few of the advantages of $\mathrm{CAD} / \mathrm{CAM}$ over conventional indirect restorative techniques. The current paper demonstrates two case reports where the restorative needs of the patient were fulfilled with the help of CAD/CAM technology.

Keywords: CAD/CAM, digitization, indirect restoration, modern computerized systems

\section{Introduction}

Increase in the incidences of dental caries is a significant worldwide oral health problem nowadays ${ }^{[1]}$. Despite the ever evolving ways of restoring the caries on proximal surface (Class II cavity), this kind of restoration still remains a tedious task for the dentists.

Difficulty arises when there is a loss of the proximal walls which leads to the loss of contact surface and open gingival embrasures. Hence, accurately restoring the proximal contacts with correct reconstruction of anatomic contour and appropriate proximal tightening is essential for periodontal health and, also to maintain dentition stability and occlusal harmony ${ }^{[1]}$.

Resin-based composite materials have been used to restore these cavities as they meet the esthetic demand of the patient ${ }^{[1,2]}$. In spite of the better esthetics, it has been proven that proximal contact of composite restorations may deteriorate overtime due to high occlusal wear, in addition to the considerable polymerization shrinkage which depends on the depth and location of the restoration ${ }^{[1]}$.

Owing to the technological advancements, ceramics have replaced composites as a restorative material in the fabrication of indirect inlays and onlays on posterior teeth because of their proven esthetic, physical and mechanical properties ${ }^{[2,3]}$.

Ceramic restorations generated with CAD/CAM technology ensure an ideal occlusal morphology and correct proximal contouring to overcome the disadvantages of direct resin composites ${ }^{[2]}$. Over the last decade CAD/CAM has grown in popularity. The technology and materials used with it are continuously evolving. Use of proper technique and attention to detail are essential in the treatment planning, tooth preparation, scanning and bonding the restoration, which ultimately results into a perfectly contoured life like restoration ${ }^{[3]}$.

In this article, we will present two clinical situations in which an indirect Lithium disilicate inlay and an inlay with cusp capping are fabricated using CAD/CAM technology.

\section{Case Reports}

\subsection{Case Report 1}

A 28 year old male patient reported to Department of Conservative Dentistry and Endodontics, Surendera Dental College and Research institute, Sriganganagar, with the chief complaint of food lodgement in the upper right back tooth region since one month. Clinical examination revealed brown to grey discoloration of the interproximal surface of tooth no.16 involving the mesial marginal ridge (Fig. 1), which was identified as Class II dental cavity. 
Tooth vitality tests revealed normal response to heat and cold. Bitewing radiograph revealed radiolucency involving enamel and dentin irt 16 (Fig. 2) indicating Class II dental cavity involving mesiocclusal surface irt16.

Patient was made acquainted with different treatment options from direct composite restorations to ceramic restorations. However, the high pulp horns, due to the young age of the patient, it was decided that in this case most important was to maintain as much tooth structure as possible. Patient requested for long lasting and esthetically proven restoration so, keeping the patient's request into consideration, it was to decide on a treatment plan that would be more long-lasting compared to various composite restorations. Also, keeping in mind the drawbacks of composite restorations, a minimal invasive approach with indirect porcelain restorations using CAD/CAM technology was decided.

Finally the CAD CAM inlay option was opted irt 16.

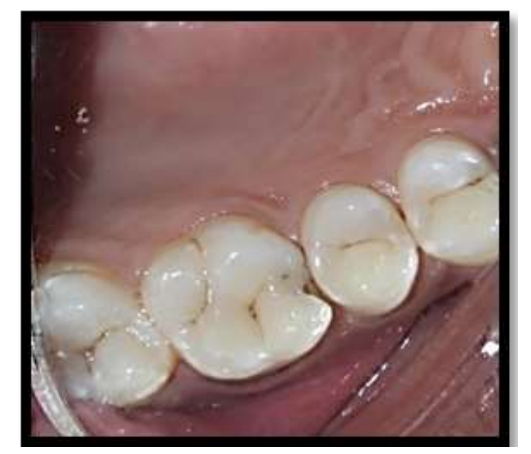

Fig 1: Pre - operative irt 16

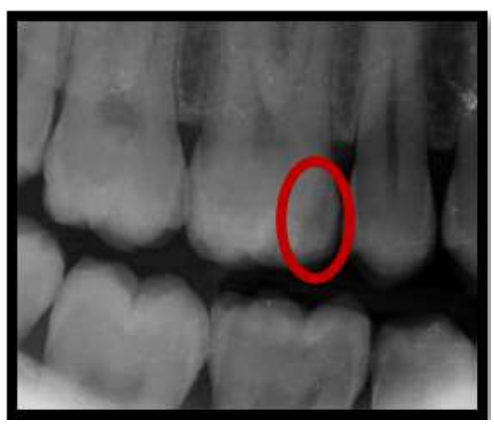

Fig 2: Pre - operative Bitewing

\subsubsection{Tooth Preparation}

After proper rubber dam isolation (Fig. 3), caries was excavated with spoon excavator (Hu-Freidy N032L DE). A Tapered Flat ended Diamond bur (Brasseler, USA) was used for tooth preparation that created occlusally divergent facial and lingual walls. Occlusally, the cavity depth was made to be $1.5-2 \mathrm{~mm}$.
Round ended bucket shaped diamond bur no. 845KR-018 (Brasseler USA) was used to shape the isthmus at the proper angulation and 849L-009 bur (Brasseler USA) was used for breaking the contacts.

Isthmus was kept at least $2 \mathrm{~mm}$ wide to decrease the possibility of fracture of the restoration. There were no undercuts that would prevent the insertion or removal of the restoration.

Facial and lingual walls were extended to the sound tooth structure, which involved the cusps in smooth curves. The facial, lingual, and gingival margins of the proximal box were extended to clear the adjacent tooth by at least $0.5 \mathrm{~mm}$. The cavity floor and its transitions with the box were rounded off and the cavity was finished with smooth and rounded edges.

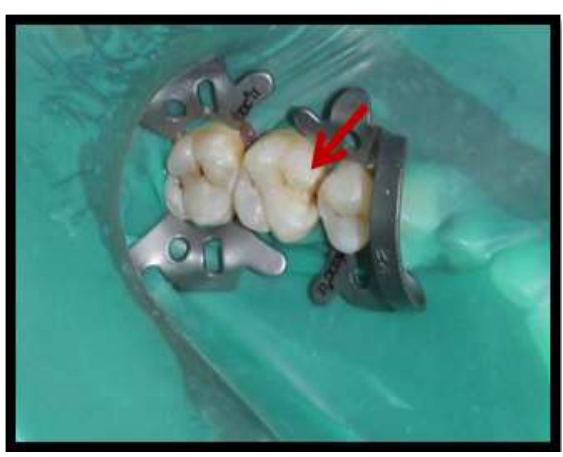

Fig 3: Isolation under rubber dam irt 16

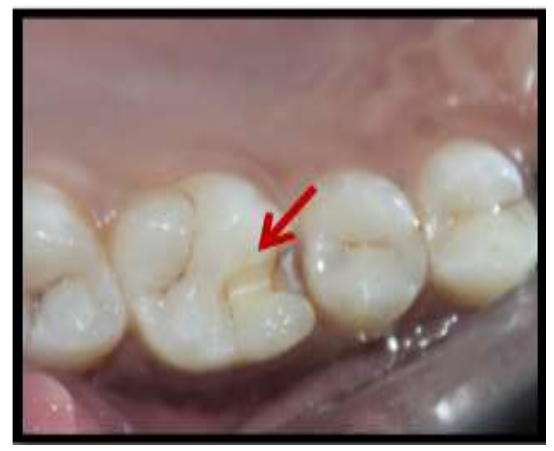

Fig 4: Cavity preparation irt 16

After evaluating the entire cavity (Fig. 4) and the interocclusal space, the impression of the tooth was taken by Double Impression technique using Addition Silicone (Aquasil LV, Putty/Light Body, Dentsply, Germany). After visualization and analysis of the quality of the impression, ceramic shade was selected using Vita shade guide 3D- master and the impression was sent to the laboratory. Inlay was fabricated in the laboratory using CAD-CAM technology and was positioned on the master cast (Fig. 5)
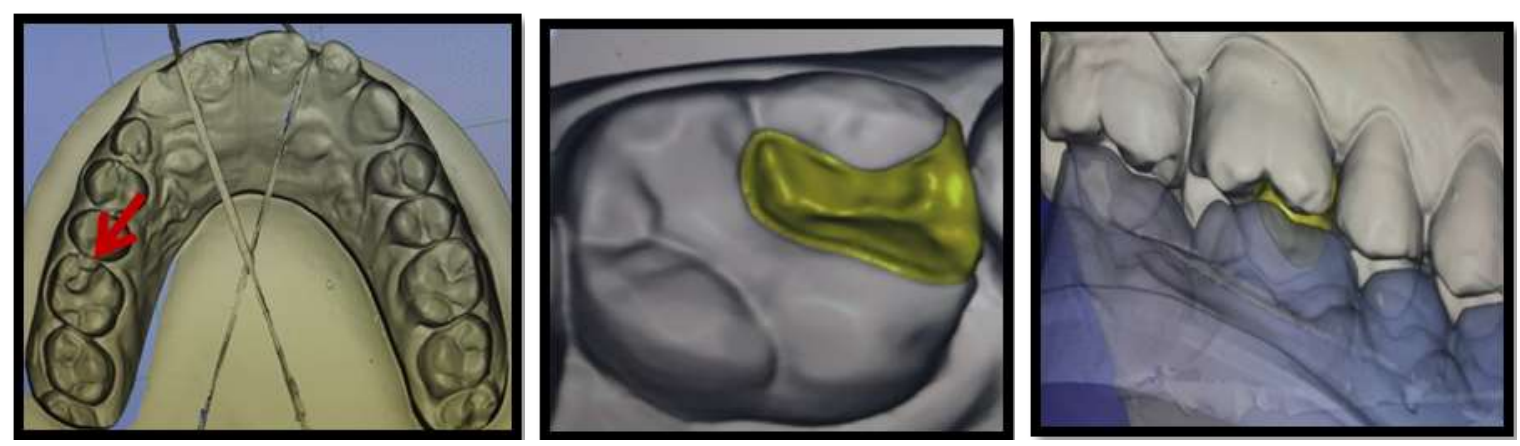

Fig 5: Showing CAD-CAM scanning and preparation 
The CEREC 3D CAD/CAM system was used for optical impression of the preparations, designing and manufacturing of the restorations (Fig. 5). IPS Empress CAD HT (A3/I12) block was used for the restoration. The chosen color was in reference to the color of the second premolar. After milling the restoration was tried in and subsequently characterized with staining and glazing.

The internal surface of the inlay (IPS Empress CAD HT) was etched with 5\% hydrofluoric acid (acid hydrofluoric ceramic etching gel®, ivoclar vivadent) for $90 \mathrm{~s}$, and then washed under running water and air dried. A silane coupling agent was applied for $3 \mathrm{~min}$, (as per the manufacturer's instructions). The tooth was cleaned thoroughly for any remnants, pumice slurry was used and the tooth was rinsed and lightly dried. For the dental substrate treatment, 37\%

phosphoric acid (N Etch $®$, ivoclar vivadent) was applied for 15 seconds followed by washing and air drying; two consecutive coats of the adhesive system ( $\mathrm{N}$ Bond Universel®, Ivoclar Vivadent) were then applied for 20 seconds then gently air-dried and light-cured for 10 seconds. Resin cement (Relyx Unicem, SPE3M E) was used as an adhesive for the cementation of the inlay. Excess cement flesh was removed and light-curing was done for 20 (s). The occlusion was checked and the restoration was finished and polished (Fig. 6). Post cementation instructions were given to the patient.

When patient was recalled after 1 year, the follow up showed that the restoration was esthetically and functionally satisfactory, without complications (Fig. 8).

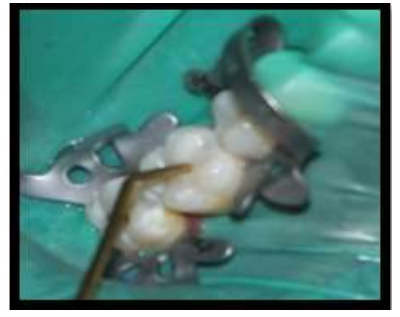

Fig 6: Cementation under rubber dam isolation

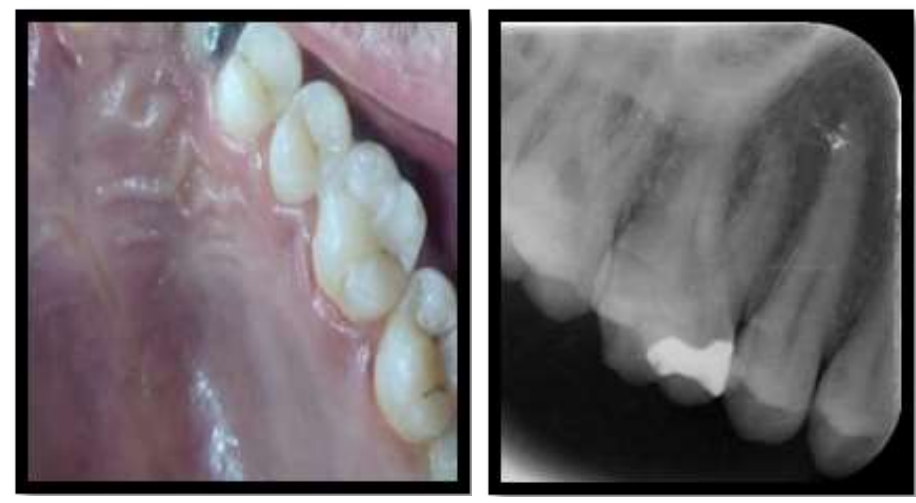

Fig 7: Post-Operative
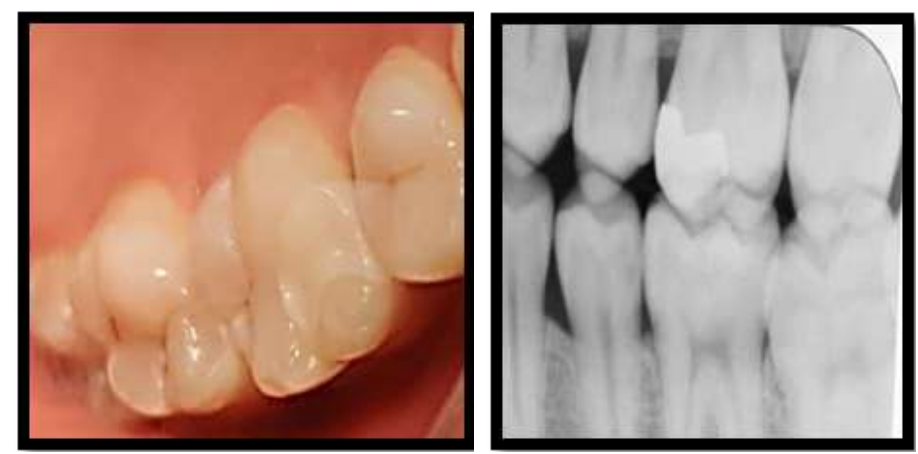

Fig 8: One Year Follow-Up

\subsection{Case Report 2}

A 32 year old male patient reported to Department of Conservative Dentistry and Endodontics, Surendera Dental college and Research Institute, Sriganganagar, with the chief complaint of blackish discolouration in the upper left back tooth region since last 1 month. Clinical examination revealed Class II dental caries involving the mesial marginal ridge of upper left molar with discolored mesiobuccal cusp irt 26. On further visual examination enamel defects were noticed on the mesiobuccal cusp of the tooth, Vitality tests revealed normal response to heat and cold.

Bitewing radiograph (Fig. 9) of the tooth revealed mesioocclusal radiolucency involving enamel and dentin irt 26.

Treatment plan of CAD/CAM processed Inlay with cusp capping was opted irt 26 owing to the patient's wide smile which placed the tooth in the esthetic zone. A minimal invasive approach was practiced to preserve the compromised remaining tooth structure with this indirect porcelain restoration. 

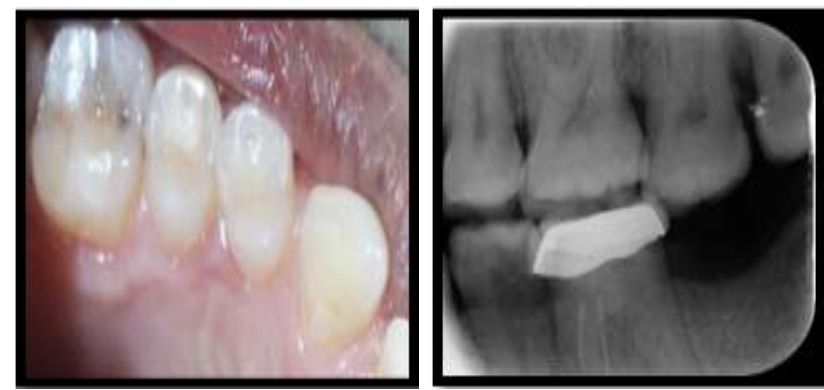

Fig 9: Pre- Operative image of 26 and bitewing radiograph

\subsection{Tooth Preparation}

A mesio-occluso-buccal (MOB) preparation was done by reducing the mesio-buccal (MB) cusp with cylindrical diamond rotary instruments with rounded angles (no. 8845 KR, no. 837 KR; Brasseler).
A conventional preparation with a modified shoulder finish line (Fig. 10) was achieved with $2 \mathrm{~mm}$ reduction of the MB cusp and a $1.2 \mathrm{~mm}$ shoulder connecting the reduced cusp with the mesial box.

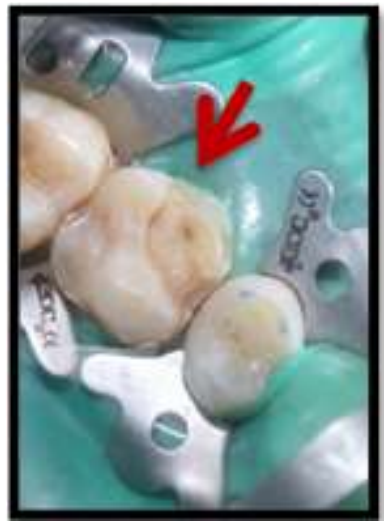

Fig 10: Isolation under rubber dam irt 26

After evaluating the entire cavity, the impression was taken by double impression technique using Addition Silicone impression material (Aquasil Lv, Putty/Light Body, Dentsply, Germany). The ceramic shade was selected and after analyzing the quality of the impression, it was sent to the laboratory.

CEREC 3D CAD/CAM system was used for optical impression of the preparations, designing and manufacturing of the restoration (Fig. 11). IPS Empress CAD HT (A3/I12) blocks were used for the restoration. Try-in of the restoration was done followed by staining and glazing.

The internal surface of the restoration (IPS Empress CAD HT) was etched with 5\% hydrofluoric acid (acid hydrofluoric ceramic etching gel ${ }^{\circledR}$, ivoclar vivadent) for $90 \mathrm{~s}$, and then washed under running water and air dried. A silane coupling agent was applied for $3 \mathrm{~min}$, (as per the manufacturer's instructions). The tooth was cleaned thoroughly for any remnants, pumice slurry was used and the tooth was rinsed and lightly dried. For the dental substrate treatment, 37\% phosphoric acid (N Etch $\AA$, ivoclar vivadent) was applied for 15 seconds followed by washing and air drying; two consecutive coats of the adhesive system ( $\mathrm{N}$ Bond Universel®, Ivoclar Vivadent) were then applied for 20 seconds then gently air-dried and light-cured for 10 seconds. Resin cement (Relyx Unicem, SPE3M E) was used as an adhesive for the cementation of the inlay. Excess cement flesh was removed and light-curing was done for 20(s). The occlusion was checked and the restoration was finished and polished (Fig. 12). Post cementation instructions were given and patient was scheduled for follow up.

At the 12-month follow up, restoration and remaining tooth structure were found to be intact and no marginal discoloration or recurrent caries was noted. The patient himself was satisfied with the treatment outcome (Fig. 13).
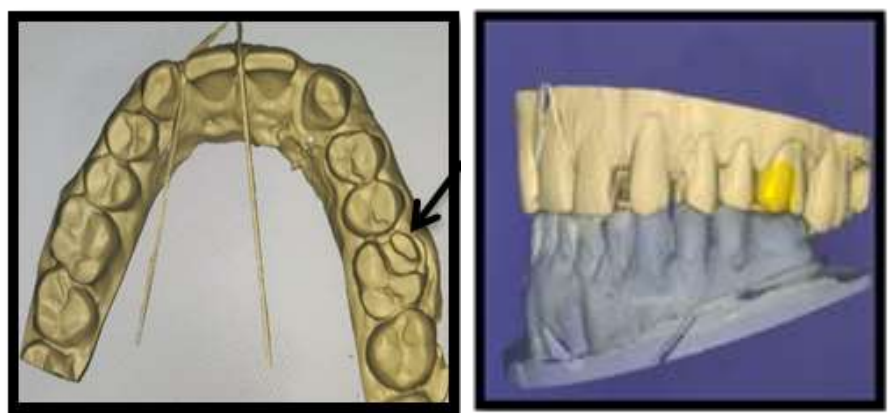

Fig 11: Image showing CAD-CAM scanning and preparation 

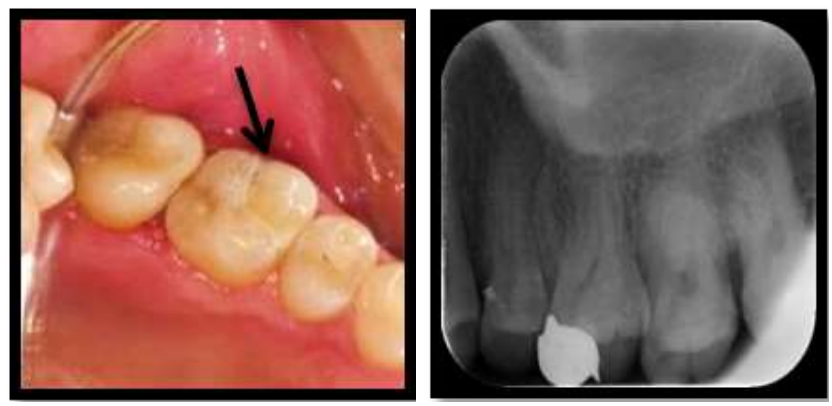

Fig 12: Post-Operative
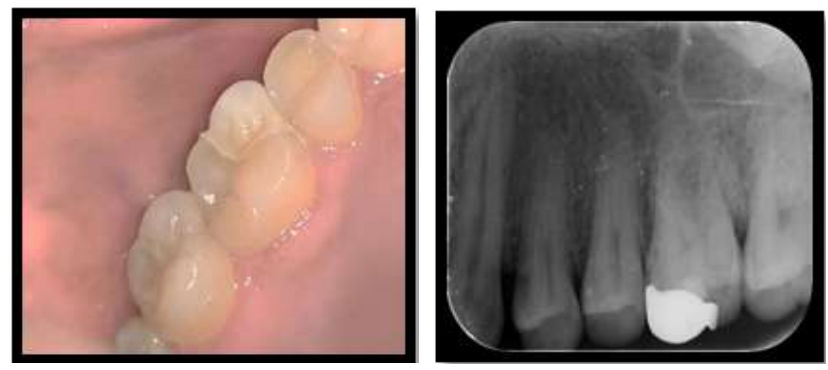

Fig 13: One year Follow-Up

\section{Discussion}

In the current scenario, treatment strategies that offer ideal mastication, as well as an esthetic perception, are deemed most satisfactory to the patient. Today's high expectations and esthetic needs, led to the emergence of Ceramics as a restorative material ${ }^{[4]}$.

Although, composite restorations have been an excellent option and are indicated for an esthetic and conservative treatment of caries, they have certain limitations, such as lack of lasting ability through years, polymerization shrinkage and inferior esthetic in long term due to stain absorption ${ }^{[5]}$. In contrast, ceramic restorations provide a better outcome due to their better stress distribution, marginal adaptation, and cusp stabilization ${ }^{[6]}$, which was the deciding factor in opting for ceramic restorations for present cases.

Moreover, the CAD/CAM technologies used in cases were to ensure an ideal occlusal morphology and optimum proximal contours to restore the teeth and to increase the longevity of the restoration ${ }^{[1]}$.

The superiority of ceramics over other restorative materials has been proven since a long time with many studies like this clinical assessment by Fuzzi et al in 1999 which proved that ceramic inlays showed a lack of recurrent caries, only slight changes in color match, and excellent longevity proved that ceramic inlays are a valuable tool for the restoration of posterior teeth ${ }^{[7]}$.

It was also supported by a clinical study by R. T. Lange which showed that indirectly manufactured ceramic inlays (Evopress) performed better than direct composite (Filtek $\mathrm{Z} 250)$ restorations in anatomic form, marginal adaptation and color match ${ }^{[8]}$.

Another study evaluated the clinical performance over the 10yrs service of class II Cerec inlays. The results revealed that the patient satisfaction and the acceptance of Cerec inlays high. The performance of these inlays after 10 years of clinical service was also acceptable ${ }^{[9]}$. This longevity influenced our decision of using Cerec system in our cases.

IPS Empress CAD systems are high performance leucite reinforced blocks for CAD/CAM technology. It shows balanced chameleon effect through its homogeneity and light scattering behavior.
Ceramic blocks possess average biaxial flexural strength of $185 \mathrm{MPa}$, along with the precise light optical properties. The restorations fabricated with these IPS Empress CAD restorations shows promising natural translucency and convincing esthetics ${ }^{[10]}$.

Various methods, for e.g., cytotoxic testing and agar diffusion testing have been used to check the biocompatibility of ceramic materials in the oral environment. Cytotoxic response of lithium disilicate dental ceramics was examined by Brackett, Wataha, and others in the year 2008. They concluded that these materials do not appear to be any more cytotoxic than other materials successfully used for dental restorations ${ }^{[11]}$.

In a three-dimensional finite element analysis study done in year 2008, it was found that ceramic inlays demonstrated better marginal integrity than the composite resin inlays ${ }^{[11]}$. Actually, the increase of the rigidity of restoration material considerably lowers the stress at the adhesive joint. The survival rate of indirect restorations have a better survival rate than direct ones. Studies reveal that, $75 \%$ of inlay /onlays are still functional after 10 years, whereas $50 \%$ of direct restorations need to be replaced at 9 years ${ }^{[12]}$.

In the presented case reports the cement used for adhesion was a self-adhesive resin cement (RelyX Unicem, 3M ESPE). Different types of resin cement that can be used in luting CAD/CAM-ceramic restorations are; 1) "etch-and-rinse" resin cements, 2) "self-etch" resin cements and 3) self-adhesive resin cements ${ }^{[13]}$.

In both these cases the enamel defects were less severe thus consequently it was considered that normal enamel could be present at the preparation sites among the affected enamel and therefore a successful adhesion could be achieved ${ }^{[9]}$. Many studies have claimed that self-adhesive resin cements present varying performances from time to time and that they are less effective compared to "etch-and-rinse" types of resin cements even though they present an acceptable behavior.

Adhesive dentistry and CAD/CAM technology has enabled us to undergo posterior indirect restorations efficiently. These restorations ensure the utmost conservation of the remaining dental structures and an efficient reinforcement of the decayed tooth. 


\section{Conclusion}

Along with the advancement of dental materials, adhesive dentistry and CAD/CAM technology has enabled us to undergo posterior indirect restorations efficiently. These restorations ensure the utmost conservation of the remaining dental structures and an efficient reinforcement of the decayed tooth. They provide us with the advantage of creation of correct anatomical contours and contact surfaces which contribute to the self-cleansable area and to the preservation of interdental papilla. Nevertheless, the success rate and durability of CAD/CAM restorations is determined through respecting the indications, the choice of the luting agent, the form of preparation and the mastery of the adhesive protocol.

\section{Conflict of Interest}

No potential conflict of interest relevant to this article was reported.

\section{References}

1. Contribution of Ceramic Restorations to Create an Optimal Interproximal Area. Journal of Clinical and Medical Images 2019;2(3):1-6.

2. Park J, Tai K, Morris J, Modrin D. Clinical Considerations of Open Gingival Embrasures 2012.

3. Weinstein G, Howard WS, Fox R. Indirect Restorations with CAD/CAM Technology 2017, 161-176.

4. Santos MJ, Costa MD, Rubo JH, Pegoraro LF, Santos GC Jr. Current all-ceramic systems in dentistry: a review. Compend Contin Educ Dent 2015;36(1):31-40.

5. Pita A, Marinou G, Tsitrou E. Case Report: Ultraconservative CAD/CAM Ceramic Restorations for the Treatment of Developmental Enamel Defects. Int J Oral Dent Health 2016;2:034.

6. Thordrup M, Isidor F, Hörsted-Bindslev P. Aprospective clinical study of indirect and direct composite and ceramic inlays: ten-year results. Quintessence Int 2006;37(2):139-144.

7. Fuzzi M, Rappelli G. Ceramic inlays: clinical assessment and survival rate. J Adhes Dent 1999;1:71-79.

8. Lange RT, Pfeiffer P. Clinical Evaluation of Ceramic Inlays Compared to Composite Restorations. Operative Dentistry 2009;34(3):263-72.

9. Sjögren G, Molin M, van Dijken JW. A 10- year prospective evaluation of CAD/CAM manufactured (Cerec) ceramic inlays cemented with a chemically cured or dual cured resin composite. Int $\mathrm{J}$ Prosthodont. 2004;17:241-246.

10. IPS Empress CAD; Ivoclar Vivadent

11. Dejak B, Mlotkowski A. Three-dimensional finite element analysis of strength and adhesion of composite resin versus ceramic inlays in molars. J Prosthet Dent 2008;99(2):131-140. doi:10.1016/S0022-3913(08)600293

12. Pallesen U, Qvist V. Composite resin fillings and inlays. An 11-year evaluation. Clin Oral Investig 2003;7(2):7179. doi:10.1007/s00784-003-0201-z

13. Radovic I, Monticelli F, Goracci C, Vulicevic ZR, Ferrari M. Selfadhesive resin cements: a literature review. J Adhes Dent 2008;10:251-258. 